\title{
LITERATURBERICHT
}

\author{
Margit Bussmann
}

\section{Quantitative Studien zu Geschlechtergleichheit und Frieden}

Forschungsarbeiten zur öffentlichen Meinung bestätigen, dass Frauen in ihren Einstellungen pazifistischer sind als Männer und im Allgemeinen zögerlicher den Einsatz von militärischen Mitteln unterstützen. Daraus abgeleitet wird die Erwartung, dass es bei einer stärkeren Beteiligung von Frauen an politischen Entscheidungsprozessen weniger militärische Konflikte geben sollte. Ein systematischer Test dieser Hypothese ist schwierig, da nur sehr wenige Frauen in politischen Positionen sind, in denen über den Einsatz von Truppen entschieden wird. Jedoch zeigen verschiedene quantitative Studien, dass Staaten mit einem höheren Prozentsatz an Frauen im Parlament friedlicher sind. Zusätzlich verweisen Studien auf eine Verbindung zwischen Geschlechtergleichheit auf gesellschaftlicher Ebene und Frieden innerhalb von und zwischen Staaten. Eine höhere Beteiligung von Frauen im formalen Arbeitsmarkt und ein besserer Zugang zu Bildung und Gesundheitsversorgung stehen in negativem Zusammenhang mit dem Ausbruch von bewaffneten Konflikten.

Schlagworte: Einstellungen, Pazifismus, öffentliche Meinung, Frauen, Frieden, politische Teilhabe

\section{Einleitung}

In der Konfliktforschung gibt es eine rege Auseinandersetzung mit verschiedenen Formen von Diskriminierung und Polarisierung als Quellen von innenpolitischer Gewalt. Der systematische Ausschluss von ethnischen Minderheiten wird nicht selten als Ursache von Bürgerkriegen hervorgehoben. Vergleichsweise wenig Beachtung fand bisher die Diskriminierung nach Geschlecht. Hinweise verdichten sich, dass es einen systematischen Zusammenhang zwischen der Genderungleichheit in einer Gesellschaft und ihrer Friedfertigkeit gibt. Erste quantitativ-empirische Studien zeigen, dass Staaten, in denen Frauen stärker politisch repräsentiert sind, aber 
sich auch vermehrt in das Erwerbsleben integrieren, weniger wahrscheinlich in militärische Konflikte verwickelt sind. Quantitative Umfrageanalysen zu politischen Einstellungen und öffentlicher Meinung deuten darauf hin, dass es systematische Unterschiede zwischen den Geschlechtern in der Beantwortung von Fragen zu Krieg und Frieden gibt. Der folgende Literaturbericht bietet zunächst einen Einblick in quantitative Studien zur pazifistischen Einstellung von Frauen und Männern und legt im dritten Teil verschiedene Erklärungsansätze für dieses Phänomen dar. Besonderes Augenmerk möchte ich dabei auf Erklärungen richten, die im Pazifismus von Frauen eine Reaktion auf die relativ stärkere Belastung, die Frauen durch Kriege erfahren, sieht. Der vierte Teil des Literaturüberblicks gilt der Frage, ob mehr Frauen an der Macht ein Weg zu einer friedlicheren Welt sein könnten. Der fünfte Teil legt die Literatur zur Integration von Frauen am Arbeitsmarkt und deren Bedeutung für friedliche Gesellschaften dar, bevor im Schlussteil Forschungslücken identifiziert werden.

\section{Sind Frauen in ihrer Einstellung pazifistischer?}

Studien der politischen Meinungsforschung bestätigen, dass es Unterschiede zwischen Männern und Frauen in ihrer politischen Einstellung gibt. Frauen sind beispielsweise in ihrer Haltung zu Globalisierung protektionistischer und unterstützen Handelsliberalisierungen weniger stark als Männer (Kalthenthaler et al. 2004: 841; Mayda/Rodrik 2005: 1402-1404). Diese protektionistische Einstellung geht auch einher mit der stärkeren Unterstützung von Frauen für eine aktivere Rolle der Regierung bei der Bereitstellung von Arbeit im öffentlichen Sektor (Iversen/Rosenbluth 2006: 14-17).

Differenzen bestehen vor allem in den Einstellungen zur Außenpolitik, insbesondere bei Fragen zu Krieg und Frieden. Frauen unterstützen im Allgemeinen den Einsatz von militärischen Mitteln weniger stark als Männer. Dabei sind die Unterschiede viel ausgeprägter, wenn die Fragestellung einen konkreten Konflikt betrifft, im Gegensatz zu Fragen zur Sicherheitspolitik hypothetischer Natur (Conover/Sapiro 1993: 1095). Dieser relative Pazifismus von Frauen zeigt sich in mehreren Ländern und Militärkrisen (Nincic/Nincic 2002: 549-551; Eichenberg 2003: 121-126), wobei es durchaus Differenzen bei verschiedenen Konflikten gibt. Eine Studie zum Zweiten Golfkrieg 1990-91 zeigte, dass die Unterschiede zwischen amerikanischen Frauen und Männern sehr groß waren (Conover/Sapiro 1993: 1091-1094), dagegen waren sie beim NATO-Einsatz im Kosovo und auch bei Fragen zur Intervention in Ruanda gering (Eichenberg 2003: 126, 130-131). Die Auswertung einer Vielzahl an Meinungsumfragen zum Einsatz der US-Streitkräfte in 
zehn militärischen Konflikten im Zeitraum 1990-2003 offenbarte, dass diese Geschlechterunterschiede nicht nur einzelne Militäreinsätze und Konflikte betreffen, sondern durchaus verallgemeinert werden können. Trotzdem findet sich eine Varianz. Vor allem scheint nicht unerheblich zu sein, wie die Frage formuliert wird. Werden bei den Umfragen in der Fragestellung die Aussicht auf (militärische und zivile) Todesopfer genannt, sinkt die Unterstützung des Militäreinsatzes zwar auch bei Männern, aber bei Frauen ist der Rückgang viel stärker ausgeprägt. Auch der Zweck des Einsatzes spielt eine Rolle. Bei beiden Geschlechtern ist die Unterstützung höher, wenn es sich um humanitäre Interventionen handelt, aber bei Frauen ist dieser Effekt markanter (Eichenberg 2003: 127-137). Während sich zwar die meisten Untersuchungen auf die US-amerikanische Öffentlichkeit konzentrieren, kommen Studien zu anderen Ländern zu ganz ähnlichen Ergebnissen (Togeby 1994: 377-380; Wilcox et al. 1996: 72-77; Bjereld 2001: 307). Zudem sind Frauen stärker als Männer gegen Folter und die Benutzung von harschen Befragungsmethoden (Haider-Markel/Vieux 2008). Insgesamt bestätigen zahlreiche Studien der politischen Meinungsforschung die These, dass Frauen pazifistischer als Männer sind. ${ }^{1}$

\section{Warum sind Frauen pazifistischer?}

Die Literatur bietet für dieses Phänomen des stärkeren Pazifismus bei Frauen verschiedene Erklärungen an. Auf biologische Faktoren Bezug nehmend werden das Sexualhormon Testosteron und das Stresshormon Cortisol in Verbindung mit aggressiven und dominanten Verhaltensweisen bei Männern untersucht (Mazur/Booth 1998). Die Aggression zwischen Männern ist in diesem Kontext relevant, insbesondere wenn Männer im Wettbewerb um Ressourcen, Dominanz oder Status stehen. Die genetische Erklärung akzeptierend schreibt Francis Fukuyama (1998: 34), dass »the basic social problem that any society faces is to control the aggressive tendencies of its young men . $^{2}$

Ein zweiter Erklärungsansatz für den weiblichen Pazifismus konzentriert sich auf die unterschiedliche Sozialisierung von Männern und Frauen und stellt Genderidentität und die unterschiedliche Verbreitung von Normen zu Gendergleichheit in den Vordergrund. Jungen werden erzogen, hart und kämpferisch zu sein, während

1 Die geringe Unterstützung bei Frauen für Militäreinsätze wurde teilweise auch mit deren geringerem Interesse an Außenpolitik in Verbindung gebracht (Togeby 1994: 380-382).

2 Die Problematik der jungen Männer taucht auch in der Bürgerkriegsforschung auf. Empirische Arbeiten zeigen, dass eine hohe Einschulungsrate männlicher Jugendlicher und eine geringe Arbeitslosigkeit eine Konflikt reduzierende Wirkung haben (Collier/Hoeffler 2004). Die hierfür angebotene Erklärung zielt darauf ab, dass junge Männer somit beschäftigt seien und nicht den Rebellen als Rekrutierungspool zur Verfügung stünden. 
Mädchen zur Empathie und Unterordnung sozialisiert werden. Feminität wird verbunden mit Gewaltfreiheit, Emotion und Harmonie und Maskulinität mit Aggression und Vernunft (Caprioli 2003: 197-199; Melander 2005; Clasen/Zwingel 2009: 134-135). Interessant ist hierzu vor allem eine Studie mit schwedischen Kindern, die zu Themen der Außen- und Verteidigungspolitik befragt wurden. Bei den Kindern (im Gegensatz zu Erwachsenen) war die Kluft zwischen den Geschlechtern bei den meisten Fragen sehr gering bzw. nicht existierend mit einer großen Ausnahme: Bei Mädchen war die Angst vor Krieg stärker ausgeprägt (Bjereld 2001: 310-312).

Eine Variante der identitätsbasierten Ansätze hebt die Mutterrolle hervor. Frauen gebären und ziehen Kinder auf und seien deswegen weniger bereit Kriege zu unterstützen, in denen ihre eigenen Kinder getötet werden könnten. Das Geschlecht von Kindern kann in der Tat einen Einfluss auf die Einstellung der Eltern haben. Urbatsch (2009) zeigt, dass bei Eltern von Jungen (ohne zwischen Vätern und Müttern zu differenzieren) die Einstellungen zur Außenpolitik pazifistischer sind, sie also Militäreinsätze weniger stark unterstützen und eher für eine Politik des Isolationismus sind, als bei Eltern von Mädchen. Urbatsch begründet dies mit der Sorge der Eltern von Jungen, dass ihre Kinder später in den Konflikt verwickelt sein könnten. ${ }^{3}$

Ein dritter Erklärungsansatz ist im Rational-Choice-Ansatz zu finden. Demnach wäre es im Eigeninteresse von Frauen, Gewalt und militärische Mittel nicht zu favorisieren. Frauen sind die Hauptopfer von Kriegen und genau darum ist es nicht in ihrem Interesse, militärische Konflikte zu unterstützen, da sie mehr zu verlieren als zu gewinnen haben. Zwar werden mehr Männer als Frauen in direkten Kampfhandlungen getötet oder verwundet, ${ }^{4}$ aber trotzdem können Frauen zwischen die Fronten geraten, besonders in Bürgerkriegen und in Konflikten mit hohem Grad an Gewalt gegen die Zivilbevölkerung. In vielen Konflikten sind Frauen von sexueller Gewalt betroffen, wenn auch in unterschiedlichem Ausmaß und aus unterschiedlichen Gründen. Frauen und Mädchen können als Sexsklavinnen für Kombattanten entführt werden, wie z.B. durch die dafür berüchtigte Lord's Resistance Army in Ostafrika. Sexuelle Gewalt kann strategisch eingesetzt werden, d.h. gezielt, um eine bestimmten Gruppe zu bestrafen oder zu verängstigen, oder aber wahllos gegen die Zivilbevölkerung, um Angst und Schrecken zu verbreiten. Sie kann auf opportu-

3 Verschiedene Studien finden einen Zusammenhang zwischen der Geburtenrate und dem Ausbruch von Konflikten (z.B. Regan/Paskeviciute 2003: 295-296). Luttwak (1995: 115) bietet eine mögliche, wenn auch nicht unbedingt überzeugende Erklärung für diesen Zusammenhang, indem er argumentiert, dass in Gesellschaften mit vielen Kindern pro Familie die Toleranz für Verluste steigt.

4 Nur in wenigen Konflikten sind Frauen in bedeutsamer Zahl aktiv an Kämpfen beteiligt. So berichtet Wood (2006) etwa von Zwangsrekrutierungen von Frauen im Bürgerkrieg in Sri Lanka. 
nistische Weise eintreten, besonders wenn entsprechende Normen und Sanktionsmechanismen fehlen, um die Kämpfer zu disziplinieren (Leiby 2009; Wood 2006).

Frauen zählen überproportional zu den indirekten Opfern. In Kriegen kann es zu Engpässen in der Versorgung mit Nahrungsmitteln und sauberem Wasser kommen, die Infrastruktur (auch im Gesundheitsbereich) wird beschädigt oder zerstört. In männlich dominierten Gesellschaften sind Frauen am Ende der Versorgungskette. Diese indirekten Konsequenzen von bewaffneten Konflikten treffen Frauen besonders hart. So haben Frauen in Friedenszeiten zwar eine längere Lebenserwartung als Männer, der Unterschied wird jedoch durch militärische Konflikte geringer (Plümper/Neumayer 2006). Vor allem wenn die langfristigen Auswirkungen von Bürgerkriegen untersucht werden, zeigt sich, dass Todesfälle und Behinderungen, die indirekt und erst nach dem Konflikt auftreten, aber auf diesen zurückzuführen sind, die Zahl der Todesopfer und Verletzten als direkte Folge während des Konfliktes übersteigen. Bürgerkriege steigern das Risiko der Erkrankung an Infektionen wie Malaria und Tuberkulose und auch die Mordrate. Zudem weisen Staaten, in deren Nachbarschaft ein Bürgerkrieg ausgefochten wird, eine gesteigerte Anzahl an AIDS-Erkrankten und Todesopfern auf. Diese Studie zeigte vor allem, dass Kinder und Frauen besonders verbreitet zu den indirekten Opfern gehören (Ghobarah et al. 2003).

Frauen werden auch aus rein wirtschaftlichen und finanziellen Erwägungen gegen Bürgerkriege sein. Frauen und Männer sind in unterschiedlichem Maße in bezahlter und nicht-bezahlter Arbeit konzentriert, haben also unterschiedliche Verbindungen mit dem Markt, was ihr Verhalten und ihre Präferenzen beeinflusst (Beneria 2003; Ruspini 2001). Durch den geringeren Zugang zu Erwerbstätigkeiten verfügen Frauen über weniger Einkommen als Männer und sind überproportional von Armut betroffen. Deswegen ist es besonders für Frauen von Bedeutung, ob ein Staat ein soziales Netz bereitstellen kann. Auch aus diesem Grund ist es plausibel, dass Frauen zögerlicher sind, Aktionen zu unterstützen, die mit einer Erhöhung des Militärbudgets einhergehen. Höhere Militärausgaben gehen auf Kosten anderer Staatsausgaben, wie z.B. Investitionen in das Bildungs- und Gesundheitswesen oder Ausgaben für die soziale Wohlfahrt, also Ausgaben, von denen Frauen stärker profitieren würden (Wilcox et al. 1996: 69).

Eine weitere Erklärung für die unterschiedlichen Meinungen bei Frauen und Männern in Bezug auf den Einsatz von Gewaltmitteln ist in der ungleichen Verteilung von Macht und physischer Stärke zu finden. Frauen sind im Allgemeinen hinsichtlich physischer Stärke gegenüber Männern benachteiligt. Daraus lässt sich ableiten, dass Frauen eine stärkere Präferenz für gewaltfreie Konfliktlösungsmechanismen haben und physischer Gewalt ein möglichst geringes Legitimationsniveau 
zusprechen möchten. Frauen haben weniger politische und soziale Ressourcen und würden verlieren, wenn Legitimität auf Gewalt und Zwangsmittel übertragen würde (Bjereld 2001: 314).

\section{Frauen in Positionen politischer Macht als Garant für eine friedlichere Politik?}

Aus den Befunden bezüglich der pazifistischen Einstellungen von Frauen nährt sich die These, dass die Welt eine friedlichere wäre, wenn mehr Frauen an die Macht gelangten. Eine angemessene Vertretung und Partizipation von Frauen an politischen Entscheidungsprozessen könnte für eine friedliche Außenpolitik ausschlaggebend sein. Die pazifistische Haltung von Frauen sollte sich in eine entsprechende Politik übersetzen, wenn wir annehmen, dass persönliche Einstellungen und Persönlichkeitsmerkmale von Herrschenden deren Politikkurs mitbestimmen (Hermann 1980).

Besonders Frauen als Staatsoberhaupt, Premierministerin oder Verteidigungsministerin sind in Positionen, in denen über die Entsendung von Truppen entschieden wird. Zwar haben Frauen verstärkt Kabinettsposten inne, jedoch häufig in Ressorts wie Gesundheit, Erziehung, Familie, Kultur und bisher selten in den Ressorts Verteidigung und Außenpolitik (Reynolds 1999: 561-564; Escobar-Lemmon/TaylorRobinson 2005: 829). Frauen sind kaum in Positionen zu finden, in denen direkt über den Gebrauch von Waffengewalt entschieden wird. Zudem werden prominente Beispiele von mächtigen Frauen nicht unbedingt mit einer friedlichen Politik in Verbindung gebracht: Margret Thatcher mit dem Falklandkrieg, Indira Ghandi mit dem dritten Indisch-Pakistanischen Krieg, Golda Meir mit dem Jom-Kippur-Krieg. Auch das von einem langen Bürgerkrieg geplagte Sri Lanka wurde lange Zeit von Frauen regiert: der Premierministerin Sirimavo Bandaraneike und ihrer Tochter, der Präsidentin Chandrika Kumaratunga, die zu Beginn ihrer Amtszeit zwar eine versöhnliche, aber dann später eine militärisch harte Linie gegen die Rebellengruppe verfolgte. Es gibt also durchaus prominente Beispiele, in denen Frauen nicht unbedingt zurückhaltend mit dem Einsatz von Waffen umgingen. ${ }^{5}$ Es gibt selbstverständlich auch zahlreiche Beispiele für regierende Frauen, die für Frieden stehen, wie etwa die frühere Ministerpräsidentin von Bangladesh, Hasina Wajed, die einen Bürgerkrieg mit einem Friedensabkommen beendete und dafür mit dem FelixHouphouët-Boigny-Friedenspreis ausgezeichnet wurde.

5 Ein gängiger Einwand ist, dass Frauen nur dann an die Spitze gelangen können, wenn sie sich der männlichen Handlungsweise anpassen. 
Um zu entscheiden, ob Frauen in der Regierungsführung im Allgemeinen für eine friedliche Außenpolitik stehen, wäre eine systematische, statistische Analyse einer größeren Zahl an Fällen notwendig, was jedoch durch die sehr geringe Anzahl an Frauen in entsprechenden Positionen erschwert wird. Deswegen weichen Studien auf die Anzahl der weiblichen parlamentarischen Abgeordneten aus. Hierbei gilt die Annahme, dass eine hohe Anzahl an Parlamentarierinnen mit der Anzahl der Ministerinnen korreliert (Escobar-Lemmon/Taylor-Robinson 2005: 835). Zudem kann sehr wohl eine starke Vertretung von Frauen im Parlament hemmend wirken, da - abhängig vom politischen System - die Entscheidung über die Entsendung der Streitkräfte häufig durch die Legislative abgesegnet werden muss.

In der Tat weisen mehrere empirische Studien auf einen Zusammenhang zwischen der Anzahl der weiblichen Abgeordneten und Frieden hin. So sind zwei Staaten mit einem höheren Anteil an Frauen in der Legislative einem geringeren Risiko ausgesetzt, miteinander in einen militärischen Konflikt verwickelt zu werden (Regan/ Paskeviciute 2003: 295-296). Dieser Zusammenhang besteht nicht nur für internationale Konflikte. Staaten mit hohem Frauenanteil im Parlament laufen zudem geringere Gefahr, in einen Bürgerkrieg verwickelt zu werden (Melander 2005 a; Bussmann 2010: 10) und sind in Bezug auf die Einhaltung von Menschenrechten erfolgreicher (Melander 2005 b), wohlgemerkt auch dann, wenn für alternative Erklärungsfaktoren (z.B. das Entwicklungsniveau oder den Demokratiegrad eines Staates) kontrolliert wird.

Nicht nur der Einfluss von Frauen auf höchster politischer Ebene ist bedeutsam. Auch ihre Mitsprache auf gesellschaftlicher Ebene und ihr Einfluss als Wählerinnen kann ein nicht unerhebliches Gewicht haben. Wenn wir davon ausgehen, dass die öffentliche Meinung eine wichtige Rolle in der Entscheidungsfindung politischer Führer spielt (Powlick 1995), sollten die Einstellungen der Frauen zu Militäreinsätzen entsprechend Gehör finden. Eine höhere Repräsentation von Frauen ist nützlich für eine stabile und demokratische Gesellschaft. Frauen haben das Gefühl, dass ihre Meinung stärker zählt und sie haben mehr Vertrauen in die Regierung, wenn Frauen in öffentlichen Ämtern vertreten sind (Atkeson/Carrillo 2007). ${ }^{6}$ Je länger Frauen bereits ihre Interessen durch Wahlen vertreten können, desto stärker sollte die Partizipation zunehmen. Eine stärkere und bereits länger etablierte Partizipation

6 Andererseits könnte die ablehnende Haltung von Frauen gegenüber Militäreinsätzen auch gerade aus der fehlenden Einbindung in wichtige Entscheidungsprozesse resultieren. Gruppierungen der Bevölkerung, die sich nicht durch das politische System vertreten fühlen, werden Politikentscheidungen nicht mittragen und fühlen sich dem System entfremdet. Darin könnte ein Grund liegen, warum Frauen solch kostspielige und riskante Unterfangen wie militärische Einsätze nicht unterstützen (Nincic/Nincic 2002: 555-557). 
von Frauen im politischen Leben könnte somit ein Hemmnis sein, Waffengewalt einzusetzen. Allerdings ließ sich bisher kein signifikanter Zusammenhang zwischen der Dauer des Frauenwahlrechts und dem Bürgerkriegsrisiko finden (Bussmann 2010:10).

\section{Sind geschlechtergleiche Gesellschaften friedlicher?}

Frauen können durch ihren Einfluss auf der gesellschaftlichen Ebene zu mehr Frieden beitragen. Frauen, die besser gebildet sind und aktiv am Erwerbsleben teilnehmen, gehen häufiger an die Wahlurne und haben insgesamt eine positive Auswirkung auf die Demokratie eines Landes (Brown 2004; Barro 1997). Mädchen den Zugang zu Bildung zu verwehren und Frauen vom formalen Arbeitsmarkt auszuschließen bedeutet auch, dass das durchschnittliche Humankapital geringer ist. Eine Geschlechterdiskriminierung im Bildungsbereich wirkt sich nachteilig auf Investitionen und Wirtschaftswachstum aus (Tzannatos 1999: 560; Klasen 2002: 362; Dollar/Gatti 1999). Ein demokratisches Regierungssystem und ein hoher Entwicklungsstand eines Landes wiederum verringern die Wahrscheinlichkeit, dass ein Staat in einen Bürgerkrieg verfällt (Hegre/Sambanis 2006).

Das Argument, das Frauenarmut mit Pazifismus verbindet, kann direkt nur schwer empirisch überprüft werden. In den meisten Staaten wird Einkommen zumeist auf der Haushaltsebene ermittelt, ohne zwischen den einzelnen Mitgliedern zu differenzieren. Es ist also kaum festzustellen, wie viel Einkommen den Frauen selbst zur Verfügung steht. Viele Studien weichen deswegen auf Bildung- oder Gesundheitsindikatoren oder auf die Erwerbstätigkeit von Frauen aus (Gray et al. 2006; Bussmann 2007). Ein Zusammenhang zwischen der weiblichen Erwerbsquote und Bildungsabschlüssen von Frauen einerseits und internationalen und innerstaatlichen Konflikten andererseits zeigt sich in verschiedenen Zeitreihen-Querschnittsanalysen (Caprioli 2003: 205; 2005; Regan/Paskeviciute 2003: 295-296; Melander 2005 a; Bussmann 2010: 10-11). ${ }^{7}$

Mit einer stärkeren Teilnahme von Frauen auf dem formellen Arbeitsmarkt erhöht sich ebenfalls ihr Einfluss. Weibliche Erwerbstätigkeit sowie die Einschulungsraten in die Sekundarstufe erhöhen den Pool von gut ausgebildeten und berufstätigen Frauen, aus dem sie in wichtige Positionen in der Legislative und Exekutive gelan-

7 Dies findet sich selbst dann, wenn alternative Erklärungsfaktoren einbezogen werden. Die Kontrolle für andere Erklärungsfaktoren ist notwendig, da die verschiedenen Befunde zu politischer und wirtschaftlicher Partizipation oder zu Wohlfahrtsindikatoren für Frauen Scheinkorrelationen sein könnten. Wichtig erscheint hier besonders eine adäquate Kontrolle für das Entwicklungsniveau von Staaten, das politische System und die Qualität der Regierungsführung (Bussmann 2010: 10). 
gen können. ${ }^{8}$ Auf politischer Ebene fallen Frauen nicht nur als potenzielle Wählerinnen in demokratischen Systemen stärker ins Gewicht, sondern auch als Steuerzahlerinnen.

Durch Berufstätigkeit verbessern sich die Autonomie und die Verhandlungsposition von Frauen in der Familie, besonders wenn es darum geht, die Haushaltsarbeiten innerhalb der Familie und Partnerschaft zu verteilen. Frauen können ihre Positionen besser durchsetzen, wenn sie sich durch eine Erwerbstätigkeit eine sogenannte Outside-Option verschaffen. Bildung und der Erwerb von Fähigkeiten sind hierbei besonders dienlich (Iversen/Rosenbluth 2006: 3-4). Frauen werden ein stärkeres Interesse daran haben, dass der Mann zuhause mitarbeitet oder einer Erwerbsarbeit nachgeht, anstatt einen Mann an ihrer Seite zu haben, der in den Kampf zieht. ${ }^{9}$ Wenn Frauen zum Haushaltseinkommen aktiv beitragen, verringert das auch den Druck auf die männlichen »Ernährer«. Sollte ein Mann arbeitslos sein, kann die Frau den Lebensunterhalt der Familie bestreiten und der Mann ist nicht gezwungen, sich dem Militär oder einer Rebellengruppe anzuschließen, um Einkommen zu erwirtschaften. Andererseits kann eine höhere weibliche Erwerbsquote zu mehr Arbeitslosigkeit bei jungen Männern führen, was den Pool vergrößert, aus dem Rebellen rekrutieren können. ${ }^{10}$

Bildung und Erwerbstätigkeit erhöhen langfristig den Status von Frauen. Ein höherer gesellschaftlicher Status von Frauen verringert nicht nur die Wahrscheinlichkeit, dass ein Land in einen Bürgerkrieg verfällt, sondern scheint auch einer anhaltenden Befriedung in Nachkriegsgesellschaften dienlich zu sein. In Gesellschaften, in denen Frauen einen höheren Status besitzen, gibt es mehr einheimische Kapazitäten und mehr Sozialkapital. Zumindest können die Fähigkeiten eines größeren Bevölkerungsteils für den Friedensaufbau genutzt werden. Eine bessere Einbindung von Frauen in den Friedensprozess und ihre aktive Teilnahme am Wiederaufbau stärkt die Rolle der Zivilgesellschaft und basisdemokratische Bewegungen. Frauen sind im Aufbau einer Unterstützung auf kommunaler Ebene für den Friedensprozess wichtig. Sie können lokale Ressourcen mobilisieren, um etwa in den Wiederaufbau von Schulen Zeit und Geld zu investieren, verfügen über wichtige soziale Netzwerke

8 Diese Hypothese fand jedoch in einer empirischen Studie Lateinamerikas keine Unterstützung (Escobar-Lemmon/Taylor-Robinson 2005).

9 Hier wird auch davon ausgegangen, dass der Mann zuhause erwünscht ist. Kalyvas (2006) berichtet beispielsweise von Fällen der Denunziation während des Zweiten Weltkrieges, bei denen die Denunziation für Frauen eine (wenn nicht sogar die einzige) Möglichkeit war, sich von ihrem Mann zu trennen.

10 Siehe Collier/Hoeffler (2004) zum negativen Zusammenhang zwischen Arbeitslosigkeit und männlicher Sekundareinschulungsrate mit Ausbruch von Bürgerkriegen. Auch Wirtschaftswachstum wird von den Autoren in dieser Weise interpretiert. 
und können beitragen, das lokale Engagement zu erhöhen (Gizelis 2009: 508-510). Im empirischen Teil zeigt Gizelis, dass UN-Friedenseinsätze in Gesellschaften, in denen Frauen eine starke Position und einen höheren Status haben, erfolgreicher sind, also zu einem länger andauernden Frieden führen. Den relativen Status von Frauen leitet sie aus der relativen Lebenserwartung von Frauen zu Männern ab. Dieser Indikator spiegelt Gendergleichheit wider und gibt Auskunft über den $\mathrm{Zu}$ gang zur Gesundheitsversorgung und anderen öffentlichen Dienstleistungen. Hier sei erwähnt, dass Frauen insgesamt eine höhere Lebenserwartung haben. Wenn also die Lebenserwartung von Frauen im Vergleich zu Männern weniger hoch ist als erwartet, zeugt das von einem geringeren Status von Frauen laut Gizelis (2009: 516-519).

\section{Schlussfolgerung}

Es gibt gute Gründe für die These, dass eine stärkere Beteiligung von Frauen am politischen, wirtschaftlichen und gesellschaftlichen Leben friedensfördernd sein könnte. In Staaten mit einer gerechteren Geschlechterordnung können Frauen besser ihre Interessen vertreten. Diese Interessen liegen stärker als bei Männern in der Vermeidung militärischer Einsätze. Dass Männer und Frauen in ihren Einstellungen zu Krieg und Frieden differieren, zeigt sich in zahlreichen empirischen Analysen. Was jedoch noch fehlt, ist eine fundierte Erklärung für dieses Muster. Erklärungen auf der Individualebene werden interdisziplinär in Zusammenarbeit mit Vertreterinnen und Vertretern der Biologie, Soziologie und Psychologie zu suchen sein, auch um methodische Fragen nach einem Genderbias in der Formulierung der Interviewfragen und dessen Auswirkungen auf die Umfrageergebnisse zu bewerten.

Auf der gesellschaftlichen Analyseebene zeigt sich ebenfalls ein positiver $\mathrm{Zu}-$ sammenhang zwischen Friedfertigkeit und Geschlechtergleichheit. Gesellschaften, in denen mehr Frauen am Erwerbsleben beteiligt sind, einen besseren Zugang zu Bildung haben, und zahlreicher in den Parlamenten vertreten sind, sind weniger wahrscheinlich der Gefahr ausgesetzt in einen Bürgerkrieg zu verfallen oder in einen internationalen militärischen Konflikt verwickelt zu werden. Mehr systematisch politikwissenschaftliche Forschung ist vor allem auf der Ebene der Gesellschaft und des Staates notwendig, um diesen Zusammenhang besser erklären zu können. Hier muss die Forschung, sowohl hinsichtlich der theoretischen Weiterentwicklung als auch der empirischen Analyse noch nachbessern. Systematische Fallstudien können bei der Entwicklung von theoretischen Erklärungen und Fundierungen hilfreich sein und Hypothesen liefern, die dann an einer größeren Fallzahl überprüft werden müssen. 
Wie in vielen anderen empirischen Studien muss die Frage nach der Richtung der Kausalität und einem Endogenitätsbias in weiteren Forschungsarbeiten aufgegriffen werden. Adäquate ökonometrische Verfahren, die explizit Endogenitätsprobleme aufgreifen, sollten zum Einsatz kommen, um zu klären, ob Geschlechtergleichheit zu mehr Frieden führt oder ob Frieden eine bessere Gleichstellung von Frauen bewirkt (Angrist/Pischke 2009; Greene 2000). Frieden kann zu mehr Gendergleichheit führen, ähnlich dem Argument, dass durch Frieden Demokratisierung begünstigt werde. Zudem bedarf die Frage, ob Friedens- oder Kriegszeiten für eine gerechte Geschlechterordnung vorteilhafter sind, einer empirischen Überprüfung. Argumente finden sich für beide Sichtweisen: In friedlichen Zeiten lässt sich die Partizipation der Zivilgesellschaft besser vorantreiben, wodurch auch Frauen mehr Mitsprache und Teilhabe erlangen. Aber es gibt auch zahlreiche Beispiele für die Gegenthese, dass Krieg für Frauen eine Chance sein kann, ihre Einflusssphäre auszudehnen. Beispielsweise bestand die erwerbstätige Bevölkerung in Deutschland 1913 zu 35\% aus Frauen. 1918 lag der Frauenanteil dann bei 55\%. Der Zweite Weltkrieg hat die Frauenerwerbstätigkeit ebenfalls in die Höhe getrieben. Auch nach Kriegsende blieb ein höherer Anteil an Frauen im formalen Arbeitsmarkt als vor dem Krieg (Wurms 1990).

Diese und weitere Fragen können durch quantitativ-empirische Studien bearbeitet werden. Nach Geschlecht unterteilte Daten zu zahlreichen Variablen sind weitläufig verfügbar. Lediglich quantitative Studien zur Analyse von Frauen in politischen Machtpositionen stoßen an Grenzen, da schlichtweg zu wenige Frauen als Präsidentin ein Land führen, um statistische Analysen mit ausreichend vielen Beobachtungen durchführen zu können. In diesem Fall sind systematische qualitative Studien eine wichtige Ergänzung. Die Fallzahl lässt sich wohl nur mit beharrlichem politischem Engagement erhöhen.

\section{Literatur}

Angrist, Joshua D./Pischke, Jörn-Steffen 2009: Mostly Harmless Econometrics. An Empiricist's Guide, Princeton, NJ.

Atkeson, Lonna Rae/Carrillo, Nancy 2007: More is Better: The Influence of Collective Female Descriptive Representation on External Efficacy, in: Politics \& Gender 3: 1, 79-101.

Barro, Robert 1997: Determinants of Economic Growth, Cambridge.

Beneria, Lourdes 2003: Gender, Development, and Globalization, New York. Bjereld, Ulf 2001: Children and the Gender Gap in Foreign Policy Issues, in: Gender \& Society 15: 2, 303-316. 
Brown, David S. 2004: Democracy and Gender Inequality in Education: A CrossNational Examination, in: British Journal of Political Science 34: 1, 137-192.

Bussmann, Margit 2007: The Effect of Trade Openness on Women's Welfare and Work Life, in: World Development 37: 6, 1027-1038.

Bussmann, Margit 2010: Political and Socio-Economic Aspects of Gender Equality and Civil War, in: Sicherheit und Frieden 28: 1, 6-12.

Caprioli, Mary 2003: Gender Equality and State Aggression: The Impact of Domestic Gender Equality on State First Use of Force, in: International Interactions 29: 3, 195-214.

Caprioli, Mary 2005: Primed for Violence: The Role of Gender Inequality in Predicting Internal Conflict, in: International Studies Quarterly 49, 2: 161-178.

Clasen, Sarah/Zwingel, Susanne 2009: Geschlechterverhältnisse und Gewalteskalation, in: Bussmann, Margit/Hasenclever, Andreas/Schneider, Gerald (Hrsg.): Identität, Institutionen und Ökonomie. Ursachen innenpolitischer Gewalt (PVSSonderheft 43), Wiesbaden, 128-149.

Collier, Paul/Hoeffler, Anke 2004: Greed and Grievance in Civil War, in: Oxford Economic Papers 56: 4, 563-95.

Conover Johnston, Pamela/Sapiro, Virginia 1993: Gender, Feminist Consciousness, and War, in: American Journal of Political Science 37: 4, 1079-1099.

Dollar, David/ Gatti, Roberta 1999: Gender Inequality, Income, and Growth: Are Good Times Good for Women?, in: Policy Research Report on Gender and Development (Working Paper Series, Working Paper No. 1), Washington, DC.

Eichenberg, Richard C. 2003: Gender Differences in Public Attitudes toward the Use of Force by the United States. 1990-2003, in: International Security 28: 1, 110-141.

Escobar-Lemmon, Maria/Taylor-Robinson, Michelle M. 2005: Women Ministers in Latin American Government: When, Where, and Why?, in: American Journal of Political Science 49: 4, 829-844.

Fukuyama, Francis 1998: Women and the Evolution of World Politics, in: Foreign Affairs 77: 5, 24-40.

Ghobarah, Hazem Adam/Huth, Paul/Russett, Bruce 2003: Civil Wars Kill and Maim People - Long After the Shooting Stops, in: American Political Science Review 97: 2, 189-202.

Gizelis, Theodora-Ismene 2009: Gender Empowerment and United Nations Peacebuilding, in: Journal of Peace Research 46: 4, 505-523.

Gray, Mark/Kittilson, Miki Caul/Sandholtz, Wayne 2006: Women and Globalization: A Study of 180 Countries, 1975-2000, in: International Organization 60: 2, 293-333. 
Greene, William H. 2000: Econometric Analysis, $4^{\text {th }}$ Edition, Upper Saddle River, NJ.

Hegre, Håvard/Ellingsen, Tanja/Gates, Scott/Gleditsch, Nils Petter 2001: Towards a Democratic Civil Peace? Democracy, Political Change, and Civil War 1816-1992, in: American Political Science Review 95: 1, 17-33.

Hegre, Håvard/Sambanis, Nicholas 2006: Sensitivity Analysis of Empirical Results on Civil War Onset, in: Journal of Conflict Resolution 50: 4, 508-535.

Hermann, Margaret G. 1980: Explaining Foreign Policy Behavior Using the Personal Characteristics of Political Leaders, in: International Studies Quarterly 24: 1, 7-46.

Haider-Markel, Donald P./Vieux, Andrea 2008: Gender and Conditional Support for Torture in the War on Terror, in: Politics \& Gender 4: 1, 5-33.

Iversen, Torben/Rosenbluth, Frances 2006: The Political Economy of Gender: Explaining Cross-National Variation in the Gender Division of Labor and the Gender Voting Gap, in: American Journal of Political Science 50: 1, 1-19.

Kaltenthaler, Karl C./Gelleny, Ronald D./Ceccoli, Stephen J. 2004: Explaining Citizen Support for Trade Liberalization, in: International Studies Quarterly 48: 4, 829-851.

Kalyvas, Statis 2006: The Logic of Violence in Civil Wars, Cambridge.

Klasen, Stephan 2002: Low Schooling for Girls, Slower Growth for All? CrossCountry Evidence on the Effect of Gender Inequality in Education on Economic Development, in: World Bank Economic Review 16: 3, 345-373.

Leiby, Michele L. 2009: Wartime Sexual Violence in Guatemala and Peru, in: International Studies Quarterly 53: 2, 445-468.

Luttwak, Edward N. 1995: Toward Post-Heroic Warfare, in: Foreign Affairs 74: 3, 109-122.

Mayda, Anna Maria/Rodrik, Dani 2005: Why are Some People (and Countries) More Protectionist than Others?, in: European Economic Review 49: 6, 1393-1430.

Mazur, Allan/Booth, Alan 1998: Testosterone and Dominance in Men, in: Behavioral and Brain Science 21: 3, 353-363.

Melander, Erik 2005 a: Gender Equality and Intrastate Armed Conflict, in: International Studies Quarterly 49: 4, 695-714.

Melander, Erik 2005 b: Political Gender Equality and State Human Rights Abuse, in: Journal of Peace Research 42: 2, 149-166.

Nincic, Miroslav/Nincic, Donna J. 2002: Race, Gender, and War, in: Journal of Peace Research 39: 5, 547-568. 
Powlick, Philip J. 1995: The Sources of Public Opinion for American Foreign Policy Officials, in: International Studies Quarterly 39: 4, 427-451.

Plümper, Thomas/Neumayer, Eric 2006: The Unequal Burden of War: The Effect of Armed Conflict on the Gender Gap in Life Expectancy, in: International Organization 60: 3, 723-754.

Regan, Patrick M./Paskeviciute, Aida 2003: Women's Access to Politics and Peaceful States, in: Journal of Peace Research 40: 3, 287-302.

Reynolds, Andrew 1999: Women in the Legislatures and Executives of the World: Knocking at the Highest Glass Ceiling, in: World Politics 51: 4, 547-572.

Ruspini, Elisabetta. 2001: The Study of Women's Deprivation: How to Reveal the Gender Dimension of Poverty, in: International Journal of Social Research Methodology 4: 2, 101-118.

Togeby, Lise 1994: The Gender Gap in Foreign Policy Attitudes, in: Journal of Peace Research 31: 4, 375-392.

Tzannatos, Zafiris 1999: Women and Labor Market Changes in the Global Economy: Growth Helps, Inequalities Hurt and Public Policy Matters, in: World Development 27: 3, 551-569.

Urbatsch, R. 2009: Interdependent Preferences, Militarism, and Child Gender, in: International Studies Quarterly 53: 1, 1-21.

Wilcox, Clyde/Hewitt, Lara Hewitt/Allsop, Dee 1996: The Gender Gap in Attitudes Toward the Gulf War: A Cross-National Perspective, in: Journal of Peace Research 33: 1, 67-82.

Wood, Elisabeth J. 2006: Variation in Sexual Violence during War, in: Politics \& Society 34: 3, 307-341.

Wurms, Renate 1990: »Krieg dem Kriege« - »Dienst am Vaterland«: Frauenbewegung im Ersten Weltkrieg, in: Herve, Florence (Hrsg.): Geschichte der deutschen Frauenbewegung, Köln, 84-118.

\section{Die Autorin}

Prof. Dr. Margit Bussmann, Institut für Politik- und Kommunikationswissenschaft, Ernst Moritz Arndt Universität Greifswald

E-Mail: margit.bussmann@uni-greifswald.de 